Frequent washing out of the frontal sinus after the operation is not desirable in most cases, although for a time it should be washed at least once daily to prevent the tube from becoming closed by desiccated secretions.

Washing out of the frontal sinus with peroxid of hydrogen, even in weak solutions, is unsafe, even where there is very free drainage.

One of the most efficient remedies for checking the suppuration is a 10 to 20 per cent. solution of argyrol.

Reinfection may occur with attacks of severe cold in the head, but the suppuration soon ceases because the free drainage is permanent.

I believe this operation is as safe as any other that has thus far been described for the treatment of suppuration of the frontal sinus. It is verv douhtful whether the burr used in this operation could cut the dura in any case, no matter how it was applied against it. Exposure of the dura in the frontal sinus would seem no more dangerous than that occurring in operations for suppuration of the mastoid, in a large percentage of which this exposure occurs.

This operation will surely establish free drainage, even in eases of empyema in which it may not be sufficient to effect a cure. In cases that do not greatly improve within a few weeks the frontal sinus should be opened externally and cleaned out. The enlarged drainage canal resulting from the intranasal opening of the sinus would allow free drainage into the nose and would lessen the danger from the external operation.

Healing is apparently as rapid after this as after any other operation on the frontal sinus. Probably 95 per cent. of all suitable cases will practically recover within from two weeks to six months.

I believe that an operation for intranasal drainage should be first chosen in practically all cases of empyema of the frontal sinus, and I believe that the operation I have described for this purpose is the easiest, both for the patient and the surgeon, and the safest of any of the operations thus far described.

34 Washington Street.

\section{PRACTICAL CYSTOSCOPY: ITS SCOPE AND LIMITATIONS.}

WITH A REVIEW OF THE PROMINENT CYSTOSOOPES OF THE PRESENT DAY.*

BRANSFORD LEWIS, M.D.

Professor of Genitourinary Surgery, Medical Department of St. Lou is University. sT. LOUIS.

The cystoscope has been so thoroughly identified with the progress made in genitourinary surgery during the past decade that it is not without good reason that the chief measure of that progress has been ascribed to this instrument.

Without going into the history of the subject, it may be said that practical, useful, modern cystoscopy had its beginning in the period of 1875-78, when Nitze presented his early models of cystoscopes. While the stamp of this parent stock is still evident in the instruments of the present day, there has been gratifying evolution from the heavy, complicated and cumbersome instruments of large caliber, hot lamps and unreliable cooling apparatus of that time, to the light and graceful ones, of wonderful precision and wide application, of to-day;

* Read by invitation before the Buffalo (N. Y.) Academy of Medicine, Jan. 7, 1908. instruments provided with brilliant lamps that do not burn, and with means for maintaining a clear field for observation and work.

Ureteral catheterization, in connection with cystoscopy, may be divided into two periods of development, namely, previous to about 1900 , and since that time. While in the ten or fifteen years previous to 1900 , catheterizing cystoscopes had been submitted by Brenner (Fig. 1), Casper (Fig. 2), Albarran, and Nitze (Fig. 3 ), it can not be said that their use was attended with the success afforded by simple cystoscopy up to that time. In the hands of experts, attemnts at catheterization gave only a modicum of success, something like 30 to 50 per cent., leaving failure stamped on too large a proportion of cases to admit this to the standing of a reliable procedure. The apparatus and methods of Pawlik and of Kelly were applicable only to females, and though admirable, were restricted in their sphere of usefulness.

During the year or two previous to 1900 , the production, in this country, of the low-tension, cold electric lamp, led to a revolution in cystoscopes that has rendered them more practical and useful to the profession, and simplified ureteral catheterization to a like degree. From the 30 or 50 per cent. of successes, the best of that period, the more modern instruments have given from 75 to 90 per cent. of successes, enabling many of those, even, who do not make a specialty of this work to avail themselves of its great advantages.

American ingenuity deserves a full meed of credit for the great progress thus recorded. Tilden Brown first demonstrated the feasibility of the double-barreled catheterizing instrument (Fig. $\dot{4}$ ), permitting synchronous catheterization of both ureters at the one sitting. ${ }^{1}$ His instrument utilized water as a distending medium. The air-cystoscope (Fig. 5) which I introduced in 1900 at the meeting of the American Association of Genitourinary Surgeons, ${ }^{2}$ although not adding much to bladder inspection, contributed largely to the reliability of ureteral catheterization by eliminating the impediments then incidental to water distension and imperfect lens systems, etc. Bierhoff's modification of Nitze's catheterizing crstoscope (Fig. 6) represents, probably, the best model of the Nitze type of cystoscope. It permits the withdrawal of the cystoscope from the bladder after double catheterization, without twistina the catheters in the way required by the European instruments, that adopt the indirect mode of catheterization.

\section{CHARACTERISTICS OF CYSTOSCOPES.}

The several European cystoscopes, excepting the Brenner, have accomplished the following objects: (a) A good right-angle view; and (b), catheterization of the ureters by the indirect method: They are the particular objects fulfilled by Albarran's, Casper's, and Nitze's newer catheterizing cystoscopes. Another form of the Nitze cystoscope, non-catheterizing, furnishes a good retrospective view for observing the prostate and vesical neck.

An interesting and ingenious cystoscope devised by Schlagintweit (Fig. 7) furnishes a retrospective view by means of a movable lens, which when drawn within the shaft (Fig. $7 \mathrm{~A})$, gives a right-angle view, but when pushed out on the side of the shaft (Fig. \% $_{B}$ ), gives the full retrospective view. While admitting the completeness of the retrospective view thus obtained, and the ingenuity of the mechanism employed, the pos-

1. Jour. Cutan. and Genitourinary Dis., 1900, p. 418.

2. Id., 1900 , p. 420 . 
sibility of disarrangement of the mechanism and "hanging" of the lens in its eccentric position, causing difficulty of extraction, etc., must occur to the conservative operator.

In 1904, with this objection in mind, I obtained a very satisfactory retrospective view in a retrograde cystoscope. This was obtained by facing a hemispherical globular lens backward, so as to gather in the rays of light coming from the direction of the operator himself ; in other words, the vesical neck and prostate, after the introduction of the cystoscope into the bladder. This is the same principle, applied in the Otis examining cystoscope (which is non-eatheterizing, and presents only the right-angle and retrospective views), and it has also been incorporated into my later, universal cystoscope.

The European catheterizing cystoscopes, with the exception of the Brenner, have usually employed the indirect method of approaching the ureteral openings (Albarran, Nitze, Casper) ; whereas, most of the American instruments (both air and water medium) have employed the direct method, after the Brenner type.

RELATIVE ADVANTAGES OF DIRECT AND INDIRECT CATHETERIZATION.

For cystoscopy, only, the right-angle lens probably shows a greater proportion of the interior of the bladder

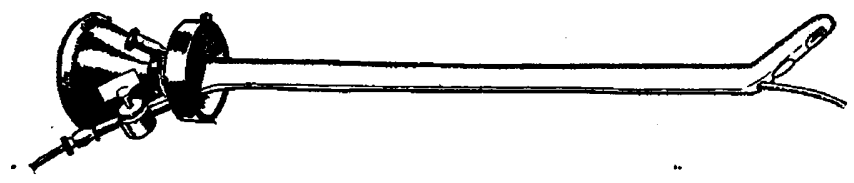

Fig. 1.-Brenner's catheterizing cystoscope.

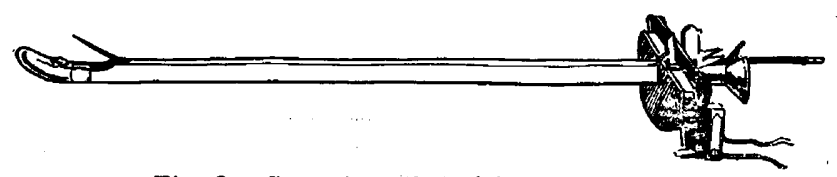

Fig. 2.-Casper's catheterizing cystoscope.

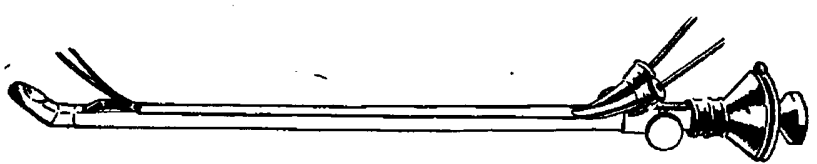

Fig. 3.-Nitze's catheterizing cystoscope.

than does the straight-view lens; but, as a matter of fact, neither one shows the whole of the interior of the organ: The right-angle lens can not show straight ahead toward the lower, posterior wall, nor can the straight-view lens show sidewise or retrospectively; and a pathologic process or growth situated at either point may readily escape detection by the lens that is inappropriate for exposing that locality. So neither the one nor the other of these two types of instrument fulfils the natural and necessary demands of complete cystoscopy.

The preference between the two types of instrument, the direct and indirect, for ureter catheterization, is also a proper subject for discussion. Under ordinary conditions and with normal bladders, the ureter openings are to be reached by either method, although there is this difference between them: In catheterizing by the direct method, the heel of the cystoscope is brought almost against the ureter opening (Fig. 8). The catheter passes out of its channel directly into the ureter opening, with scarcely any space to bridge over or prove a source of uncertainty and difficulty. When the indirect method is employed, the catheter emerges from the concavity of the cystoscope, a point which can not be brought against or even very close to the ureter opening; hence, more or less space must be bridged before the catheter reaches the opening (Fig. 9). A soft or flexible catheter is not the steadiest material one could desire under such circumstances. Another element of uncertainty is added in the movable lever for controlling the direction of the catheter. This is Albarran's device, now used on all cystoscopes that catheterize by the indirect method. From these considerations, it is probable that more skill and practice are required to execute harmoniously the several maneuvers incident to carrying out the indirect method than is true of the direct method. Abnormal conditions may alter the verdict.

Ordinarily, by elevating the outer end of the directview cystoseope, there is no difficulty in bringing the ureter openings into view. for catheterization (Fig. 8), but occasionally a "precipitate" bladder is met with (Fig. 9), in which the ureter orifices are placed below a jutting prostate and outside of the field of vision of the direct-view lens. Under such circumstances, the
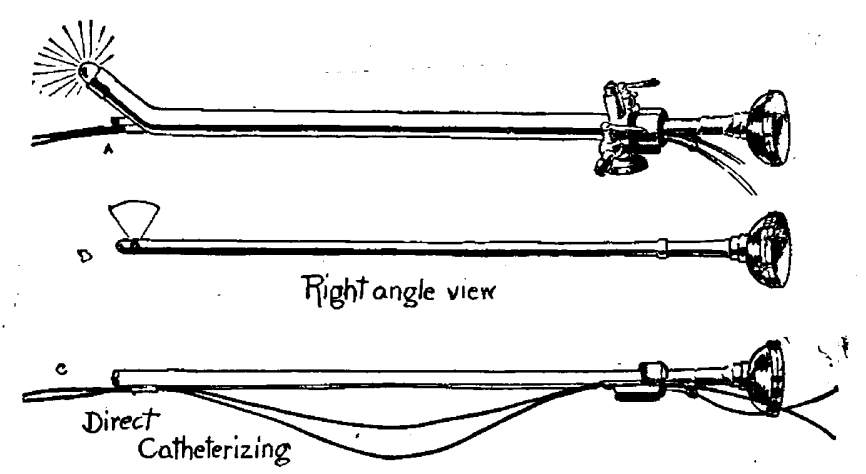

Fig. 4.-Tilden Brown's double-barreled catheterizing cystoscope, permitting synchronous catheterization of both ureters at one sitting.

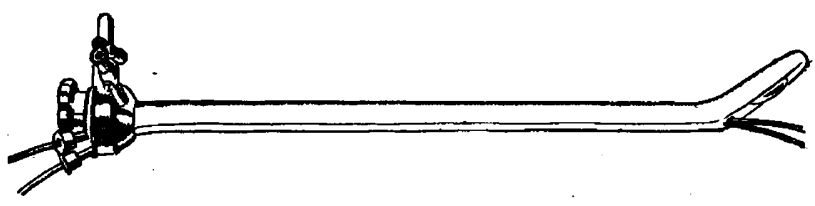

Fig. 5.-Bransford Lewis' air cystoscope.

indirect method is much more appropriate, and may, in fact, readily overcome the difficulty, or may be the only practicable route to the ureteral openings. It is again apparent, therefore, that one type of instrument alone can not meet the requirements of all the varying conditions encountered in the clinic.

\section{AIR AND WATER AS DISTENDING MEDIA.}

There has been much discussion as to whether air or water is the better medium for bladder distension in cystoscopy. My own views on the subject have undergone considerable change in the past few years, influenced, largely, by overcoming some of the difficulties that were formerly connected with the use of water in this capacity.

It can not be denied that water is the medium most suited to the bladder, and the one to which it has been accustomed all its life, so to speak; while air is distinctly irritating and very disagreeable to the bladder, although not dangerous, as has been claimed by some of our German confrères. Moreover, water is the better 
medium for exhibiting papillomatous or fimbriated growths in the bladder. It floats their filmy strands in full and natural view before the eye, while air causes them to collapse in a mass against the bladder wall.

It is evident, therefore, that in ordinary cystoscopy and ureteral catheterization, if the difficulty due to hemorrhage and pus clouding the distending medium can be overcome, water is decidedly the medium of choice. This difficulty has been overcome. The universal cystoscope permits of rapid and effective change of fluid, even during the work of the operator. The large lumen of the sheath, through which the exchange of fluids is made, amply takes care of this feature. Consequently, for these two purposes, cystoscopy and ureter catheterization, air inflation has been practically discarded by me.

With respect to operative cystoscopy, however, other factors have a bearing and lead to a different conclusion. Then, it is not so much a question of the comfort of the patient (which can be taken care of by some form of anesthesia) as it is expediency in attaining the object to be accomplished, the removal of a growth by a snare; the extraction of a calculus from the lower end of a ureter; the dilatation or cutting of a strictured ureteral

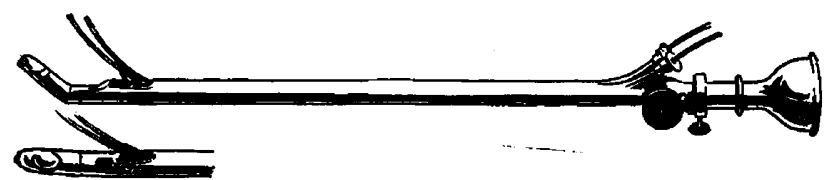

Fig. 6.-Bierhoff's modification of the Nitze catheterizing cystoscope.
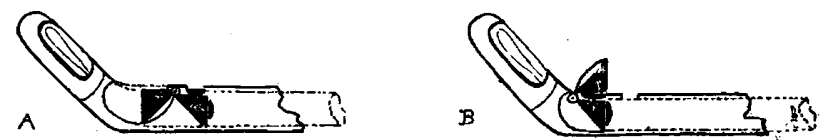

Fig. 7.-Schlagıntwelt's cystoscope. A, right angle view. B, full retrospective view.

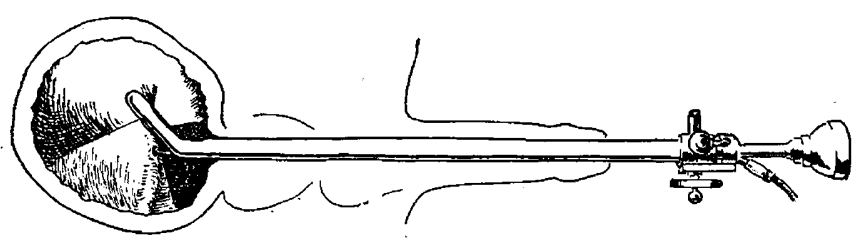

Fig. 8.-Dilect ureter catheterization.

orifice, etc. Hemorrhage, sufficient to interfere, is much more liable to occur in connection with such manipulations as these than in connection with simple cystoscopy or ureter catheterization.

For these reasons, air is the medium employed with my operative cystoscope, while water is used altogether with the universal cystoscope.

\section{A UNIVERSAL CYSTOSCOPE.}

Impressed with the shortcomings of the cystoscopic armamentarium, overlapping here and proving deficient there; the disappointments involving my own early instruments, as well, I began, about four years ago, the endeavor to develop a cystoscope that would embrace in a single instrument the several features demanded for effective and comprehensive cystoscopy, together with ureteral catheterization; embodying, also, means for adequate irrigation and rapid exchange of fluids. Practical use of the instrument (Fig. 10) during the past two years has proved that the following purposes have been amply fulfilled by it:
(1) Direct, forward view; (2) right-angle (lateral) view; (3) retrospective view; (4) double, synchronous catheterization of the ureters by the direct method; (5) the same, by the indirect method ; $;^{3}$ and, finally, (6) an irrigation feature so satisfactory that it is commonly employed in preference to a catheter for washing out the bladder preliminary to cystoscopy ; and, as previously mentioned, irrigation can be carried on during the working of the operator.

In the production of the instrument, the inestimable value of simplicity and freedom from complications has been constantly kept in mind. Problems of reliable insulation, of setting the cold lamp to best advantage, of protecting it from possible breakage, especially while within the bladder, and yet exposing its rays to both the convex and concave aspects, for illumination of the whole interior of the bladder; of reducing the outside caliber of the sheath to the minimum selected (No. 23, French), and yet permitting its carrying large enough telescopes to provide plenty of light and wide fields of vision-have engaged attention.

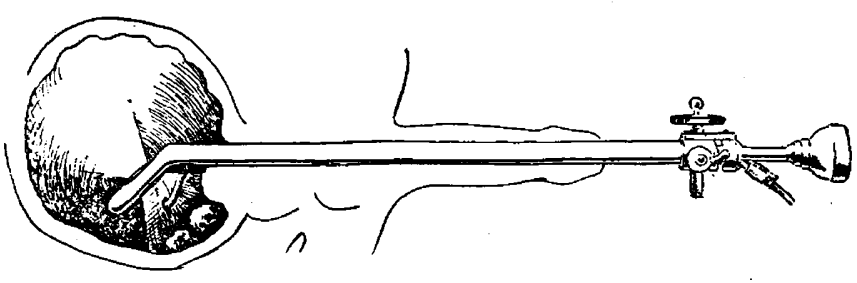

Fig. 9.-Indirect ureter catheterization.

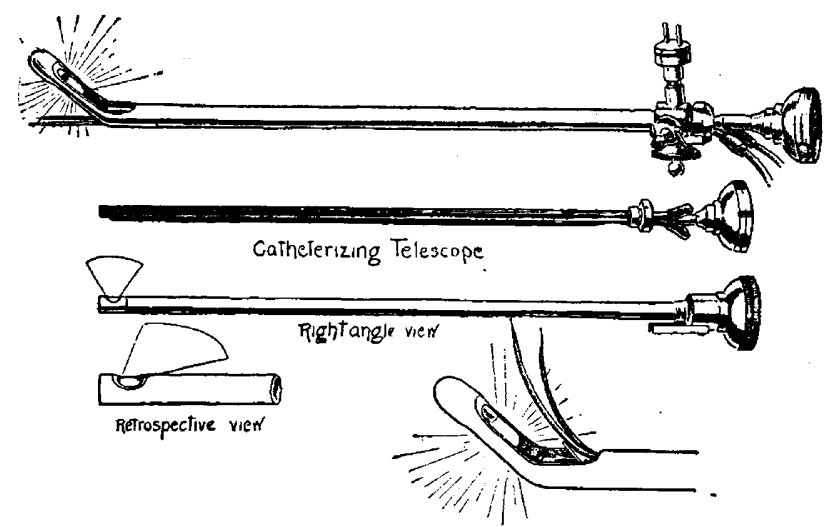

Fig. 10.-Bransford Lewis' univer'sal cystoscope.

DESCRIPTION OF THE UNIVERSAL CYSTOSCOPE.

Employing an idea first submitted by Boisseau du Rocher ${ }^{4}$ in 1890 , a service sheath was utilized to carry an insulated wire, with a cold lamp in its beak. $\Lambda$ s it was contemplated to view every point in the bladder with this instrument, the beak was fenestrated on its concave and convex surfaces, the lamp being set base upward and bulb pointing downward, shedding its best illumination in the directions most needed.

The ocular and catheterizing apparatus is embodied in telescopes that are passed freely through the sheath, so that, after the sheath is once inserted into the bladder, it remains there until all manipulations are ended, the exchanges of telescopes being made thereafter, with the sheath in place, and without the patient's noticing it. The sheath is the irrigating channel already alluded to. Water is injected through it either directly or through one of the cocks at its side.

3. Details and working model completed, but not yet on the market.

4. Ánn. d. mal. d. org. génlto-urin., 1890, 1892, 1894. 
While two different telescopes for the lateral and retrospective views are provided, experience has shown that the lenses embrace such a wide field that both views may be combined in the one telescope, which is both right-angle and retrospective. The same lens is also used with the indireet catheterizing telescope (Fig. (10). The direct catheterizing telescope supplies a good direct view, besides its catheterizing feature, and may be used for simple cystoscopy without catheterization, the catheter tubes being plugged at their outer ends to prevent the escape of the distending fluid through them. A larger, direct lens is furnished without the catheterizing feature.

TECHNIC OF CYSTOSCOPY AND URETERAL CATHETERIZATION.

It is probable that, with a good and efficient cystoscope, the greatest source of trouble or difficulty in cystoscopy arises from insufficient anesthesia. But that is

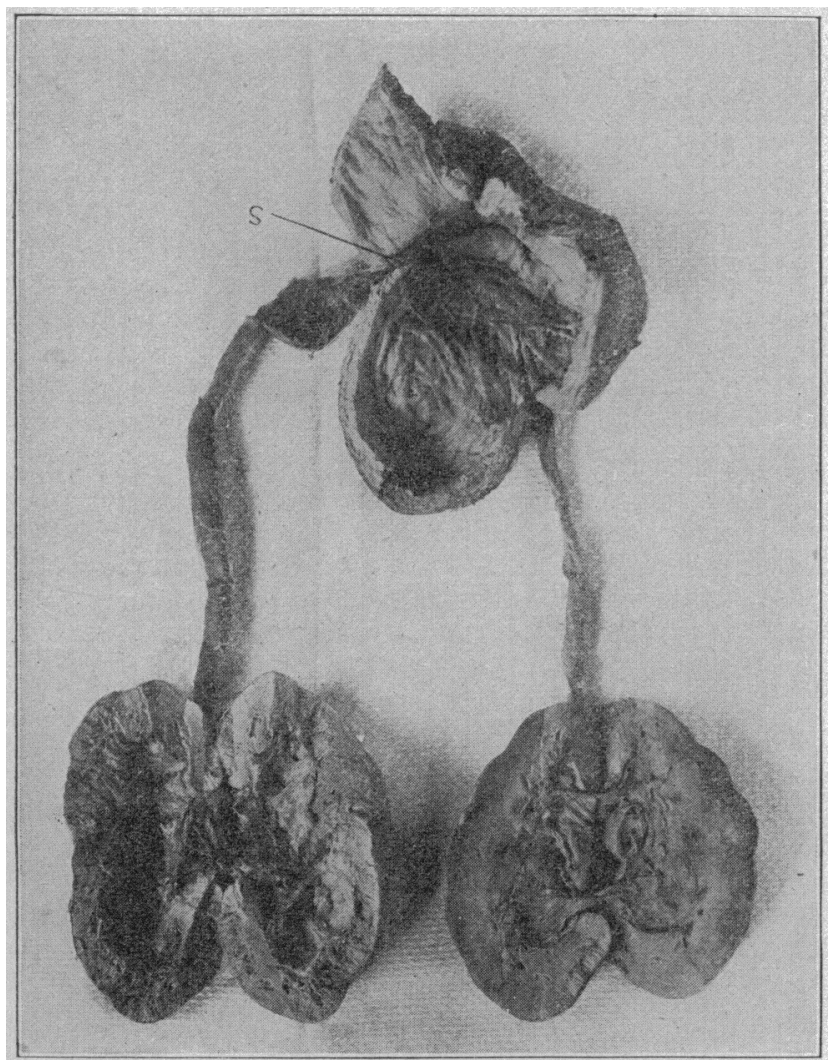

Fig. 11.- Postmortem specimen, showing a ureteral stricture which caused a dilated ureter, with subsequent destruction of the kidney, uremia and death of patient.

not to be interpreted as meaning that general anesthesia is frequently necessary. On the contrary, general anesthesia is very seldom found to be necessary for either cystoscopy or ureteral catheterization, local anesthesia, when properly and adequately administered, sufficing for the vast majority of all cases. In a number of cases it. is not necessary to use even a local anesthetic, although I deem it undesirable and injudicious to subject a patient to a painful experience when it can be made a comfortable one without adding to the risk of the procedure.

During the past two years I have practically discarded cocain in my work of this kind. I now secure local anesthesia by the use of alypin tablets, deposited at the points of election by means of my urethral tablet depositor (Fig. 13). These tablets are made of appropriate size by the Schieffelin Company and contain 11/8 grains of the drug each. In applying them the following plan is adopted: The patient first empties the bladder, or it is emptied for him by catheter, and washed with boric solution and again emptied. The depositor is inserted. as far as the posterior urethra (the anterior urethra does not usually require anesthesia), where it is held while the obturator is withdrawn; a tablet is dropped into the depositor and shoved into the urethra by means of the obturator. After macerating in the mucus of the urethra a minute or so the tablet becomes a soft mass, which is then smeared over the adjacent membrane by to-and-fro motion of the depositor. Precise information can be obtained as to the disappearance of sensitiveness by inquiry of the patient. From two to four tablets may be used, according to necessity, in the indi-

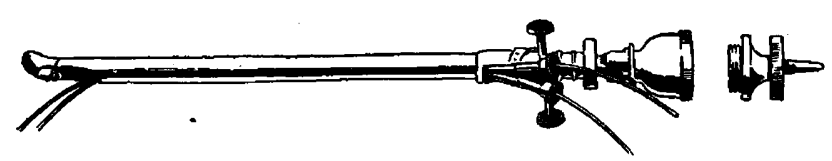

Fig. 12.-Freudenberg's catheterizing cystoscope.

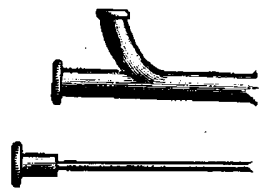

Fig. 13.-Author's urethral tablet depositor.

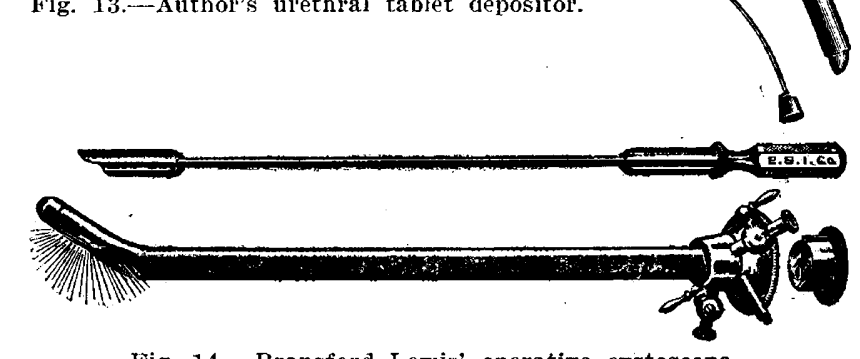

Fig. 14.-Bransford Lewis' operative cystoscope.

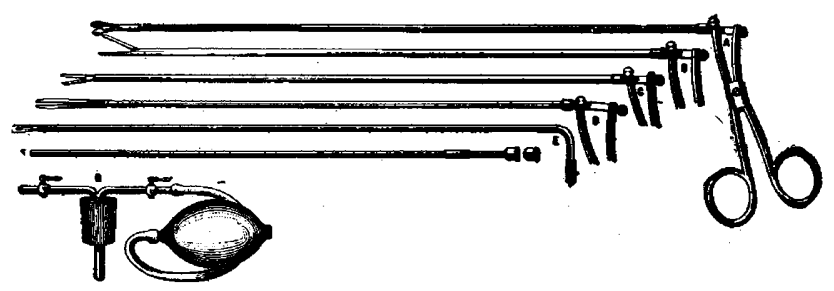

Fig. 15.-The accessories to Dr. Iuwis operative cystoscope.

vidual case, and manipulations should not be begun until acute sensitiveness is reduced.

\section{POSTURE.}

While several different positions are employed by different operators, I prefer the dorsal, with the legs supported by knee-crutches. With an extremely sensitive or a contracted bladder it is often of service to relieve the pressure of the fluid from the most sensitive area, the neck, by moderate pelvic elevation. Flexion of the thighs on the abdomen relaxes abdominal pressure and resistance, under similar circumstances.

After preliminary adjustment and turning off of the light the cystoscope is introduced into the bladder, five or six ounces of clear, warm water are injected through the sheath and allowed to flow out again. This is repeated as often as is necessary to clean the bladder, six 
or cight ounces being left in for distension. The telescope first selected is run through the sheath, the light turned on and the observation is made. The cystoscope is moved about freely and turned on its axis, no danger coming from touching the walls of the bladder with a lamp that is only moderately warm. On finishing with the telescope first used, it is replaced by another in the manner previously mentioned, and thus the entire inner strface of the bladder, including the neck and prostatic outgrowths, tumors, calculi, etc., come within the olscrvation of the investigator.

If, however, it is the intention to catheterize the ureters, too much time must not be spent in looking at the bladder before proceeding to the catheterization; the disappearance of anesthetic effect may be followed by resistance or bladder contractions that disarrange and hide the ureteral openings, possibly impeding or defeating the catheterization until renewed anesthesia is secured. The cathcterization itself is accomplished in the manner usually described, there being nothing peculiar to this instrument, except that either the direct or the indirect method may be used. In order to insure their remaining within the ureters on the withdrawal of the cystoscope it is advisable to push the catheters from six to cight inches up into the canals, but when a ureter stone or a stricture is suspected they are sent up as far as the kidney pelves.

The urine drainage that ensucs is steady and measured if the eye of the catheter is within the pelvis, but interrupted or comes in successive jets if lower down in the ureter. It is thus influenced by the peristaltic contractions of the ureter.

Some hemorrhage may become manifest in the urine drained if the catheter is roughiy introduced or is pressed too far into the pelvis. When this is casual, and without material bearing on the diagnostic evidence, the red blood cells may quickly be cleared from the urine by adding acetic acid to the slide on which the sediment is placed. This not only removes the red cells, but clears up the nuclei of pus and renders the epithelium distinct.

MODE OF WITHDRAWING THE CYSTOSCOPE AND LEAVING THE CATHETERS IN PLACE.

(A) After direct catheterization; (B) after indirect catheterization.

(A) The water that distends the bladder is first allowed to escape through one of the cocks; the light is turned off and the cord disconnected. While feeding the catheters into the channels the cystoscope is, at the same time, drawn outward; when the beak end emerges from the meatus the catheters are grasped at that point and held while the instrument is being removed. Another mode is to withdraw the catheterizing telescope from the sheath until the catheters are exposed just beneath the shoulder, near the ocular end; there they are grasped and held while the telescope is removed, after which the sheath is in turn removed.

(B) After indirect catheterization the mode of removal is essentially the same, except that the cystoscope must be turned on its axis before withdrawal to bring the concavity forward in harmonv with the natural curve of the urethra. This brings about crossing of the cathetere within the bladder, and the extraction is not accomplished as easily as after the use of the direct nrode; nevertheless, by care and practice it is accomplished. If found impossible in a given case the drainage may be secured before removal of the cystoscope.

Tnder ordinary circumstances cystoscopy and ureteral catheterization may be carried out in a well-equipped office, the patient suffering practically no pain and experiencing no ill effects afterward. It has been thought by some that bladder segregation of the urine is a simpler and less painful procedure. My own estimate, made after use of both methods, is that segregation, in addition to being of little reliability in diagnosis and of none in therapy, is really more arduous and painful than is ureteral catheterization and removal of the cystoscope.

\section{LIMITATIONS OF CYSTOSCOPY.}

Like every other surgical procedure, cystoscopy has its limitations. It can not be used, for instance, where there are urethral strictures too narrow to permit of the introduction of a cystoscope; although, of course, this is a matter in which the caliber of the cystoscope has a bearing. I have one cystoscope, caliber 18, French, which passes through a pretty small urethra. The caliber of the universal cystoscope is 23, French. The calibers of late European cystoscopes, as advertised in the Iatest catalogues of European makers, are, respectively, Nitze, 25; Kollman, 26; Wossidlo, 25, French. Large prostates sometimes render the introduction of a cystoscope difficult, but it is very unusual that cystoscopy is prevented for this reason.

It has becn taught that the use of the cystoscope should be avoided in cases of urinary tuberculosis; that such instrumentation is liable to increase the seriousness of the situation. My experience leads me to believe that this is purely a priori reasoning, and, moreover. incorrect. Dr. Willy Meyer ${ }^{5}$ expresses a similar belief. I have obscrved many cases of urinary tuberculosis in which, whether because of the cystoscopic and other local measures repeatedly made use of, or in spite of them, their progress has been that of extremely gratifying improvement or even definite recovery. And this to a degree of frequency that would not be expected from the older writings advocating the "expectant" or do-nothing plans. In tuberculous pyelitis, besides ureteral lavages with boric or other mild antiseptic solutions, I have injected iodoform oil through the catheters directly into the pelves and ureters, with beneficial effect. I can not agree that urinary tuberculosis proscribes the use, to a reasonable extent, of the cystoscope.

The limitations that were formerly imposed on the cystoscope by active hemorrhage have been practically removed in the manner already described. Since the universal cystoscope has been at my disposal I have not met with a case of vesical or ureteral hemorrhage sufficiently active to prevent its satisfactory use, either for cystoscopy or ureteral catheterization, although we have made use of it in carcinomatous cases, in which a continuous stream of blood was plainly visible, coming from some point within the bladder.

Objection has from time to time been made to ureteral catheterization on the ground of supposed danger of carrying infection from the bladder up into the ureters or pelves. Ground for this objection has been denied by all writers who have had large experience in this work. Considering the great number of instances in which ureteral catheterization is being carried out all the time, if there were any material evidence in support of the claim it would have been brought forward long since.

Pain has been done away with as militating against cystoscopy, as indicated in previous remarks. It is

5. New Yolk Med. Jour., April 27, 1907. 
talien care of, if excessive, by either local, spinal or general anesthesia.

Contracted bladder is not now so great a hindrance to cystoscopy as it formerly was. With two ounces of fluid, or even one ounce, sufficient space is given to make some use of the direct-view telescope. and possibly, also, of the indirect, in certain cases; and the cold lamp does not involve the danger of burning, even with so iittle fluid. Besides, the newer, wide-angle lenses do not require the distance for focusing that was formerly nccessary to include a good field of vision.

\section{AN OPERATIVE CYSTOSCOPE}

In connection with this subject it may not be amiss to present an instrument which as yet has not become widely familiar to the profession, although I have been making use of it since 1902-03, when the first model was completed for me. I refer to my operative cystoscope (Fig. 14). A detailed report of work with it was made before the Mississippi Valley Medical Association, Oct. 11, 1904. ${ }^{6}$

While it is possible that water might be used as a distending medium for it, I have always used air, for the reasons already indicated. This instrument is mainly a straight tube, without lens system, an ocular window serving to retain the air in the bladder, and at the same time permitting the passage of the several operative instruments through the small perforation at its lower edge. Air is pumped in through a cock, by means of a double rubber bulb, and warmed air is obtained by sucking it from above an alcohol flame. In the newest model a small channel runs along the lower wali of the cystoscope to take up, by aspiration, the excess of fluid that may collect in the bladder, this tube being connected with a vacuum bottle by rubber tubing. This excess fluid is also taken care of by posture, the patient being placed in a position of exaggerated pelvic elevation or in the knee-chest position.

The appurtenances supplied with the instrument are: Alligator forceps for intra-vesical work; flexible and inflexible forceps for ureter work; scissors; dilators of different kinds; probes, sounds, bougies, catheters, electrodes, for ureter work; knife, cautery and platinum loop, etc.

While this instrument has not so wide a scope nor so frequent application as the universal cystoscope, it, nevertheless, has a definite position in vesical and ureteral work that is highly desirable. With its assistance and without the necessity of opening the bladder to direct attack, small foreign bodies, calculi etc., may be removed; strictured ureter openings are cut or dilated with as definite precision as when the same measures are accomplished in the urethra; calculi, hung in ureteral orifices, are capable of removal by means of the forceps that are manipulated under the definite observation of the operator. The flexible ureter forceps, probably the most difficult of the appurtenances to perfect, is capable of passing one or two inches up into a ureter, where it may be opened and closed in the endeavor to grasp a stone that may be incarcerated there.

Granted that this operative set may not always accomplish all that could be wished of it, it will fulfil the objects required for a number of cases at least. It has already done that for me. And failure with it does not leave the patient any worse off than before, nor in-

6. Report on Operative Work in the Ureter through the Author's Catheterizing and Operative Cystoscopes, Lancet-Clinic, Cincin. March 4, 1905 . terfere with the adoption then of the radical, cutting operation, that may prove necessary. Numerous instances are reported in which the bladder has been opened to effect the removal of a stone projecting from a ureteral orifice; others in which the same radical measure has been undertaken to permit access to a strictured ureter opening. I believe it would be a rare exception in which these objects could not be accomplished with this instrument and its appurtenances, without resorting to such radical preliminaries. Figure 11 shows a postmortem specimen of a ureteral stricture, which had produced a dilated ureter, pelvis and destruction of the kidney, uremia and death, all of which might have been obviated had the patient submitted to investigation and treatment before he was in extremis; but that was the state in which he entered the hospital, dying immediately afterward. The merest incision of the narrowed orifice, made through the cystoscope before the serious involvement of the kidncys, would probably have given him a number of years of life, if it had not reclaimed him to health.

In cheering contrast to this is presented the $x$-ray demonstration, following catheterization of the ureters with catheters containing lead-wire stylets, of an individual possessing three independent ureters, ${ }^{7}$ two of them healthy, the third infected with gonococci, the source of a recurrent gonorrhea for over five years, notwithstanding his active pursuit of relief at the hands of many practitioners during that time. Through the means mentioned, the anatomic anomaly was demonstrated; the physiologic phenomenon of supplying three different urines by one individual (differing in specific gravity and other physical characteristics), was proved; and through medicated lavage of the infected ureter, the source of infection was removed, and the patient permanently relieved. He has since married and has been healthy for over two years.

In further contrast to the lethal outcome of ureteral stricture, so graphically depicted in the postmortem specimen (Fig. 11), I would briefly mention the case of a female patient who was reduced from health to invalidism by recurrent attacks of ureteral colic, which became so severe as to require morphia and energetic sedative means. Cystoscopy led directly to the diagnosis of. stricture of the right ureter, the contraction involving the lower end of the channel. Nothing could at first be introduced, but repetition of endeavors with a variety of ureter probes and sounds finally gained access, which was followed by dilators, until this ureter outlet became as large as its companion and gave free escape to the urine. The first urine drained from the affected ureter contained much pus, some of it collected into clumps that, by plugging the narrowed outlet, had been as effective as calculi could have been for causing recurrent colics and loss of health. The outcome of such a condition, unrelieved, could only have been the destruction of the corresponding kidney, with ultimate involvement of the opposite one, whereas, the opening up of the channel by means of the operative cystoscope was followed by complete relief from suffering, the clearing of the urine and the regaining of health, weight and strength. She has been well and free from the attacks now for four years.

This case, and others that might be mentioned, are adduced not in justification of the cystoscope, but merely in illustration of the great service it is capable of rendering in the diagnosis and therapy of genito-

7. Med. Rec., Oct. 6, 1906. 
urinary affections. The cystoscope needs no justifica$t$ on at my hands. It has an established position in the esteem of the profession, from which it can not be dislodged. But, while a large proportion of the profession are arailing themselves of its assistance in both of these capacities, it is probable that a larger proportion are still viewing it rather as an attractive toy- to be admired when exhibited, but of less practical utility than enthusiasts would have them believe. And we still occasionally hear the sage advice given to determine the presence or absence of stone in a suspected kidney by exploratory nephrotomy and renal palpation; or the same means is advised for determining which is the more affected of two tuberculous kidneys, calmly jgnoring the fact that the information on such matters given by ureter catheterization, drainage and urinalysis, is far more valuable; indicating, not how a given kidney may feel, but, what is far more important, its functionating capacity.

We still, sometimes, hear of the worse of two diseased kidneys being removed because the operator, neglecting ureter eatheterization, has depended on symptomatology and exploratory palpation to guide him; when we know that the symptomatology of renal discases is notoriously misleading, utterly failing in some cases of completely destroyed kidneys, or reversed in others, so that the painful symptoms abound on the healthier side and do not show on the side affected. These and other well known vagaries of renal symptomatology should prevent undue reliance being placed on it; and, at the same time, impel us to resort to complcte and full physical examination in all such cases, the most important feature of which is the use of the cystoscope and ureteral catheterization.

\section{SCOPE OF URETERAL CATHETERIZATION.}

As indicative of the large sphere of usefulness and the wide range of information given by ureteral catheterization, alone, excluding that furnished by the cystoscopic views in the bladder, I may quote from a previous paper on the subjects as follows:

The purposes of ureteral catheterization are two-fold, for diagnosis and for treatment.

For Diagnosis.-(A) 'To locate the origin of pus, blood, tuberculous products or bacilli, the various pyogenic infections, abnormally desquamated epithelium, etc., as to whether they come from (1) the bladder, (2) the right ureter. (3) the left ureter, (4) the right kidney, (5) the left kidney, (6) the right or ( 8 ) the left peri-renal space, communicating with the corresponding kidney or ureter.

(B) To recognize and locate obstructive conditions in the right or left ureter from (1) stricture, (2) stone, (3) adjacent tumor, (4) bend or kink in the ureter from movable or dislocated kidney, (5) valvular junction of the ureter with its pelvis.

(C) To determine (1) the number of kidneys present; (2) if only one, which is absent.

(D) To determine the number of ureters present.

(E) To determine the functional activity of each kidney separately and relatively, with respect to its excretion of urea, albumin, quantity of urine, the specific gravity, etc.

(F) To determine the size and capacity of each kidney-pelvis, with respect to (1) hydronephrosis, (2) pyonephrosis, (3) total obliteration of secreting tissue.

8. Ureter Catheterism: Its Purposes and Practicability, Ann. Surg., January, 1903.
(G) If there be kidney discase present, to determine (1) if only one kidney is affected or both; (2) if only one, which is the affected one; (3) if both, which is the one more affected; (4) if removal of the worse one be advisable, is the other one able to carry on kidney functionation sufficiently; (5) if removal of one be advisable and the other is capable of supporting life. will the operation remove the infection from the body, removing the possibility of dissemination or recontamination?

For Treatment.-(A) To enlarge narrowings or strictures at (1) the ureter openings, or (2) in the channels at any point; (3) by facilitating drainage through the increased urcter-caliber thus obtained, to assist in the improvement of prelitis or pyonephrosis, unilateral or bilateral.

(B) To irrigate and medicate (1) the ureters, the kidney-pelves, of one or both sides.

(C) To assist, by anesthetizing, lubricating or enlarging a ureteral orifice, the passage through it of a calculus or plug of pus, blood, etc.

(D) To use the ureter, alter it is catheterized, as a guide in certain abdominal and pelvic operations.

(E) By prolonged catheterization and drainage of a ureter, to facilitate the cure of ureteral fistula.

\section{DIAGNOSIS OF URETERAL CALCULI.}

We often hear discussions on the respective merits of ureteral catheterization and radiography for determining the diagnosis of stone at some point in the ureter or pelvis. Impartial analysis of the conditions prevail ing leads to the belief that the two methods should go hand-in-hand, each being complementary to the other, and neither being used to the exclusion of the other. Radiographs may show shadows from phleboliths, enteroliths or other foreign substances in the line of the ureters, so that it is always incumbent on the operator to determine by catheterization whether such a foreign body be within the ureter or not, and also whether it is causing renal disturbance, as attested by the urine drawn from the same source. On the other hand, a catheter meets with an obstruction in the ureter of a urinary patient, and it often requires the interpretation of the radiogram to determine whether the obstruction be from stone or kink or other causc. Such radiograms should be taken with the catheters in the urcters and loaded with fuse-wire, as suggested by Kolischer and Schmidt.

PHYSICIANS, SURGEONS AND CYSTOSCOPES.

While it can not be expected that every one who practices medicine or surgery must adopt the cystoscope into his personal use, devoting the time necessary to beconie skilled in its use, the same thing might be said with respect to the ophthalmoscope, the stethoscope or other appliances pertaining to special ficlds of work. As physicians and surgeons, we can not avoid the rosponsibility of securing the benefits of all such means to our patients, cither by personal application or through others especially skilled in their use. It is gratifying to know that in our progressive country, and in this progressive age, there is hardly a town of any size to be found in which there is not a cystoscopist of sufficient ability and skill to give the service desired. The full force of this remark may not appear unless we revicw the instances, unfortunately not few in number, in which patients have drifted from bad to worse in urinary afflictions, soothed along, from month to month, on diuretics and "urinary tonics," so-called, nothing of real merit being 
done either in the way of diannsis or therapy, "because," as the doctor naively explains, "I haven't any rstoscope, and wouldn't know how to use it if I had one." And the patient's opportunity for reclamation to health is wasted on such flimsy grounds! I now have under observation ( $I$ can offer them nothing better) four cases of carcinoma of the bladder, in which srmptoms of the trouble had existed from three to fire years before any definite plan for diagnosis or treatment was arlopted. Each was fed on internal medicines and urinary "soothing syrups" until the condition became inoperable and plainly boyond the reach of any material aid.

1050 Century Building.

\section{HARE-LIP AND CLEF'T PALATE.}

\section{ALEXANDFR IICGH FERGUSON, M.D.} (IIICAGO.

A common deformity the surgcon is called on to correct is hare-lip, and not infrequently hare-lip and cleft palate are combined. While the operation to correct hare-lip is comparatively simple, so far as technic is concerned, much depends on carrying out the few necessary details with wreat precision and care. In cleft palate the conditions rary so much that it is impossible to make use of any one procedure that has been devised to correct this deformity in all cases. The case must be studied carefully and then the surgeon must decide which.operation is the most suitable one to employ.

'To make clear a few principles involved in both these operations I shall consider the two conditions separately.

\section{ITARE-LIP.}

Clinical Varieties. - The defect may be (1) unilateral; ( 2$)$ bilateral; (3) median; $( \pm)$ complicated by cleft palate, single or double; and (5) later, where the fissure extends up to the eye, ete.

Time to Operate. - In uncomplicated cases of harelip, no matter what the nature of the defect, the operation should be done soon after birth, on the same day, it possible. 'The impaired prehensile powers of the in. fant prevent its taking nourishment properly, and thus lead to malnutrition and non-development. If the infant is too fecble to permit of an operation, a competent nurse should be placed in charge, one who understands fecding and diet, so that the operation can be Ione inside of two or three weeks, under favorable conditions. It is also wise to defer operation until after the period of dentition. For this reason parents should bo urged to have the operation done before the tceth begin to erupt, but if centition has begun, then wait until the teeth have erupted.

When the harc-lip is complicated by cleft palate the time for repairing the lip must depend on the degree and kind of bone deformity present. Surgeons are not in accord on this point. My own practice is to restore the normal shape of the alreolar processes first, and at the end of a week to operate on the lip. I find that when the lip is sutured over a premaxilla that was wired to the superior maxillæ, and in the cleft between them, a week previously, great strength and protection is given the bony framework, and the tendency for the premaxilla to become loosened is overcome.

Principles Involved. - Although cheiloplasty is a minor operation, it is essential, as stated before, to observe carefully certain fundamental principles. These involve (1) denudation, (2) coaptation, (3) retention, (1) conformation, and (5) cleanliness. The edges of the cleft inust be freshened without removing any tissue, so as to leave broad surfaces which favor more accurate coaptation. This is done best by making flaps from the edges of the cleft, swinging then backward. and then coapting them. Economy of tissue is to be aimed at in all procelures carrjed ont to remedy congenital defects. The broader the surfaces for coaptation, the more certainly will primary union take place. Coaptation must be perfect, and retention by sutures accurate and without tension.

The objective point should be the formation of a normal upper lip, so as to obliterate even the suggestion of the pre-existing deformity. The nature of the deformity often is such that this object is defeated, but the surgeon should exercise his ingenuity to the utmost so as to do the best he can under the circumstances.

While absolute surgical cleanliness can not be attaincd in operations on the lip, the results are best when asepsis and anticepsis are carried out rigidly.

\section{THE FENGER OPERATION.}

The late Dr. Christian Fenger devised a most excellent operation for hare-lip, ${ }^{1}$ the principles of which I carry out in my practice. These are: (1) The removal of no tissue; (2) the approximation of wider raw surfaces than in the older operations; (3) the application of three rows of sutures, (a) skin, (b) mucous membrane, and (c) tension sutures.

The incision, in both single and double cases, was made at the junction of the skin and mucous membrane, imitating in this particular the flap-splitting perineorrhaphy of Lawson Tait. The two flaps are made to turn posteriorly and coaptation of the raw surfaces is accomplished by means of retention and skin sutures (Figs. 1 and 2). Dr. Fenger always used silk for all his sutures, but I prefer horsehair for the skin and retention sutures, using for the latter two hairs in each suture. Fenger also applied a strip of adhesive plaster, extending from car to ear, to relieve tension. If there is any doulst as to whether or not there is tension, it is best to scpurate the lip from its attachments on each side until tension is relieved. I do this by thrusting a sharp periosteal clevator or a pair of scissors through a small incision under the lip, close to the gum, at a little distance from other wounds, and then elevating the cheek from the bone.

In addition to these measures I employ oxid of zinc adhesive plaster as follows: Take a strip of plaster one inch wide and about twelve inches long, place the center of the strip beneath the chin, pull firmly on both onds, allow the plaster to adhere to both cheeks woll back, and then cross the plaster over the nose on to the forehead. Where the plaster passes over each eye, roll the strip on itself into a cord. This effectually prevents tension on the sutures, even when the child is crying. The line of incision should never be sealed either with collodion or plaster.

\section{THE NOSTRIL.}

It is exceedingly difficult, but none the less essential, for cosmetic purposes, to construct a normal nostril. To do this one must do an osteotomy on the side opposite the cleft. To make the deficient nostril of the same size as its fellow, it is a good plan to insert into the nostril a sound of the same size as the naris and then suture the nostril over this. If the cartilage of the

1. The Joursal A. M. A., Aug. 1. 1891, p. 176. 